\title{
Polypectomy: No training, No cancer prevention!
}

\section{(웅 $\circledast$}

Authors

Cesare Hassan ${ }^{1}$, Alessandro Repici²

Institutions

1 Endoscopy Unit, Nuovo Regina Margherita Hospital, Rome, Italy

2 Endoscopy Unit, Humanitas University, Rozzano, Milano, Italy

submitted 23.11.2016

accepted after revision $\quad 30.12 .2016$

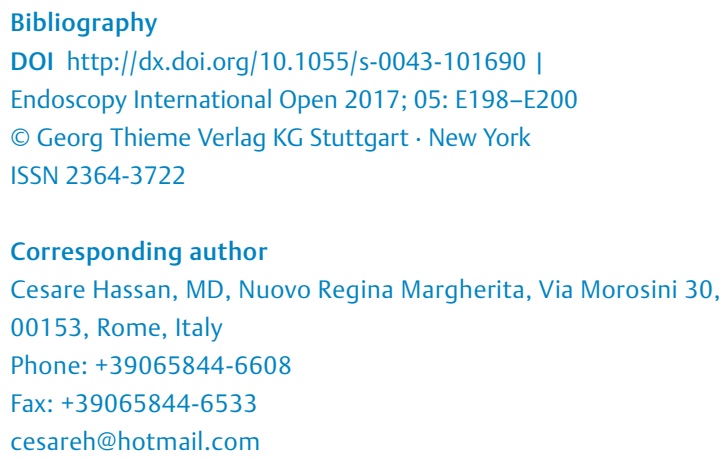

Following the publication of the National Polyp Study [1] showing the efficacy of polypectomy in preventing colorectal cancer (CRC) incidence and 2 subsequent prospective series showing the feasibility of colonoscopy as primary screening technique $[2,3]$, over $60 \%$ of the American population and $10 \%$ to $20 \%$ of the European population have undergone colonoscopy for screening purposes $[4,5]$.

The strict association between the diagnostic performance of the individual endoscopist, assessed by adenoma detection rate (ADR), and the risk of post-colonoscopy interval cancer led to the general conviction that the degree of protection following colonoscopy screening could be affected by its technical performance $[6,7]$. This was confirmed by the high variability in ADR among different endoscopists in similar or equivalent settings, as well as by the identification of relevant predictors of that variability, such as withdrawal time, level of cleansing, and expertise of the endoscopist $[8,9]$.

This evidence led to the analogy between airline companies and endoscopy services. As the former succeeded in minimizing the risk of accidents by implementing solid policies involving extensive pilot training and retraining, similar policies have been proposed for individual endoscopists performing screening colonoscopies. For instance, short retraining courses mainly focusing on lesion recognition and withdrawal technique resulted in long-term improvement in technical performance as measured by ADR, which in turn is likely to reduce risk of post-colonoscopy interval CRC [10]. Similarly, implementation of split-dose regimen resulted in a substantial increase in ADR [11]. In addition, assessment of competence of senior endoscopists based on direct observation by expert endoscopists has become a prerequisite to practice within the United Kingdom (UK) population-based screening system [12].

When considering technical competence as the main driver of CRC prevention, training is critical. To grade trainee competence, a paradigm shift from the simplistic minimum number of procedures to a more structured system, based on objective and articulated assessment of competence, has been proposed. In detail, the assessment of competency in endoscopy (ACE) system implemented in the United States is based on a complex assessment of cognitive and motor skills during 5 consecutive endoscopies after each 50 colonoscopies of the trainee. Improvement in the ACE score has been associated with a higher proficiency in the main quality indicators, such as cecal intubation rate and polyp detection rate $[13,14]$. Similarly, an assessment tool based on direct observation (DOPS) of the trainees can reassure the medical and non-medical community that only trainees with adequate skills will perform endoscopy without supervision in routine practice, reducing the variability in technical performance.

However, there are still 2 main pitfalls in training policies. First, formal training programs, based on objective assessment of competence, are lacking in several countries, especially in Europe. This may be at least partially explained by the lack of robust literature validating such programs. For instance, none of these training modules was validated in a randomized setting. Second, these programs mainly deal with the diagnostic phase of colonoscopy, with less or no focus on endoscopic resection. On one hand, this is expected, when considering the relevance of the learning curve in cecal intubation rate for trainees, due to both the complexity of the insertion phase and the clinical importance of a complete examination. In addition, competence in diagnosis may be assessed on consecutive colonoscopies, simplifying the integration between training and daily routine. On the other hand, endoscopic resection (i.e., polypectomy and endoscopic mucosal resection [EMR]) represents a crucial step in efficacy and safety of colonoscopy, as incomplete resection has been strictly associated with the risk of interval CRC [15], and most major adverse events associated with colonoscopy (i.e., bleeding and perforation) are actually related to endoscopic resection rather than to the diagnostic phase of colonoscopy

In this issue of Endoscopy International Open, Patel K et al. report on an international survey of how training and competence assessment in polypectomy are performed [16]. Overall, 610 endoscopists (57\% trainers, $43 \%$ trainees) from 20 countries addressed general training in polypectomy, as well as 
specific programs for more advanced interventions, such as resection of large polyps or mucosectomy [16].

The picture that emerged from the survey is quite dismal. First, trainers in most countries reported lack of specific national guidelines on this issue. This underscores that competence in polypectomy has not been incorporated in the professional standard for the endoscopist, leaving exploitation of formal international training programs to the willingness of individual trainers rather than to a nationwide approach.

Second, most trainees reported lack of training for polypectomy of large polyps as well as in endoscopic mucosal resection (EMR). This is quite disturbing for several reasons. First, the rate of incomplete polypectomy appeared to be strictly associated with lesion size. In particular, the rate has been reported to be nearly $20 \%$ for experienced endoscopists [17]. Thus, higher rates due to inadequate training may have worrisome consequences for cancer prevention, especially because these lesions usually represent the most advanced step of the adenoma-carcinoma sequence. Second, lack of training in EMR may result in an inability to remove large flat lesions, such as granular- or mixed-type lateral spreading tumors (LST). That may help explain why too many patients with benign LST amenable of endoscopic resection are still referred for unnecessary surgery.

Third, only half of the trainees reported systematic use of some type of formal assessment for polypectomy competence, making it likely that rates for such an assessment are very low outside UK and the United States. That is a significant issue, when considering that, unlike with diagnostic colonoscopy, polypectomy requires individual assessment of several sequential phases, such as assessment of the lesion, positioning of the scope, choice of prophylactic actions (i.e., submucosal injection, loop, etc.), selection of the device, type of current, and assessment of post-polypectomy scar. This is very well summarized in the Directly Observed Polypectomy Skills (DOPyS) tool, recently validated in the UK [18].

The main limitations of the survey by Patel $\mathrm{K}$ et al. are represented by the selection bias of the operators who replied to the survey, and by a possible heterogeneity in participation among individual countries, so that the survey may not fully represent the balance in training policies among the countries surveyed [16]. However, these limitations are likely to skew the data toward a more favorable picture, in that endoscopists who and countries that have an already established specific interest in polypectomy training were more prone to responding to the questions. In addition, such selection bias seemed to more frequently affect the trainers rather than the trainees, with the former describing a more gloomy scenario, which underlines the importance of specifically including the trainees in such surveys, as was done by the authors.

Overall, the data by Patel $\mathrm{K}$ et al. show an inadequate and fragmented policy of polypectomy training in most of the countries, which is likely to result in suboptimal performance by post-training endoscopists. That is more alarming when considering that adoption of mass population screening programs is resulting in an enormous increase in the number of colonoscopies performed, prompting employment of new endoscopists to manage to the rising number of colonoscopies. The only positive news, if any, is that the lack of adequate training programs leaves significant room for improvement, which should spur international societies to quickly implement policies aimed at selection, adoption, and further validation of training programs. In that regard, the favorable experiences that have already been documented in the United States and UK may be a reasonable starting point.

\section{Competing interests}

None

References

[1] Winawer S], Zauber AG, Ho MN et al. Prevention of colorectal cancer by colonoscopic polypectomy. The National Polyp Study Workgroup. . N Engl J Med 1993; 329: 1977 - 1981

[2] Lieberman DA, Weiss DG, Bond JH et al. Use of colonoscopy to screen asymptomatic adults for colorectal cancer. Veterans Affairs Cooperative Study Group 380. . N Engl J Med 2000; 343: 162 - 168

[3] Imperiale TF, Wagner DR, Lin CY et al. Risk of advanced proximal neoplasms in asymptomatic adults according to the distal colorectal findings. N Engl J Med 2000; 343: 169-174

[4] Stock C, Haug U, Brenner H. Population-based prevalence estimates of history of colonoscopy or sigmoidoscopy: review and analysis of recent trends. Gastrointest Endoscop 2010; 71: 366-381 e362

[5] Joseph DA, King JB, Miller JW et al. Prevalence of colorectal cancer screening among adults-Behavioral Risk Factor Surveillance System, United States, 2010. MMWR 2012; 61: Suppl 51-56

[6] Kaminski MF, Regula J, Kraszewska E et al. Quality indicators for colonoscopy and the risk of interval cancer. N Engl J Med 2010; 362: $1795-1803$

[7] Corley DA, Jensen CD, Marks AR et al. Adenoma detection rate and risk of colorectal cancer and death. N Engl J Med 2014; 370: 1298 1306

[8] Zorzi M, Senore C, Da Re F et al. Quality of colonoscopy in an organised colorectal cancer screening programme with immunochemical faecal occult blood test: the EQuIPE study (Evaluating Quality Indicators of the Performance of Endoscopy). Gut 2014; 64: 091389-1396

[9] Barclay RL, Vicari J], Doughty AS et al. Colonoscopic withdrawal times and adenoma detection during screening colonoscopy. N Engl J Med 2006; 355: 2533-2541

[10] Coe SG, Crook JE, Diehl NN et al. An endoscopic quality improvement program improves detection of colorectal adenomas. Am J Gastroenterol 2013; 108: 219-226; quiz 227

[11] Radaelli F, Paggi S, Hassan C et al. Split-dose preparation for colonoscopy increases adenoma detection rate: a randomised controlled trial in an organised screening programme. Gut 2017; 66: $270-277$

[12] Barton JR, Corbett S, van der Vleuten CP. The validity and reliability of a Direct Observation of Procedural Skills assessment tool: assessing colonoscopic skills of senior endoscopists. Gastrointest Endoscop 2012; 75: $591-597$

[13] Sedlack RE, Coyle WJ. Assessment of competency in endoscopy: establishing and validating generalizable competency benchmarks for colonoscopy. Gastrointest Endoscop 2016; 83: 516-523 e511 
[14] Sedlack RE, Coyle WJ, Obstein KL et al. ASGE's assessment of competency in endoscopy evaluation tools for colonoscopy and EGD. Gastrointest Endoscop 2014; 79: 1 - 7

[15] Farrar WD, Sawhney MS, Nelson DB et al. Colorectal cancers found after a complete colonoscopy. Clin Gastroenterol Hepatol 2006; 4: $1259-1264$

[16] Patel K, Rajendran A, Faiz O et al. An international survey of polypectomy training and assessment. Endosc Int Open 2017; 05: 190-197
[17] Pohl H, Srivastava A, Bensen SP et al. Incomplete polyp resection during colonoscopy-results of the complete adenoma resection (CARE) study. Gastroenterology 2013; 144: 74 - 80 e71

[18] Gupta S, Bassett P, Man R et al. Validation of a novel method for assessing competency in polypectomy. Gastrointest Endoscop 2012; 75: $568-575$ 